\title{
Tracking ultrafast solid-state dynamics using high harmonic spectroscopy
}

\author{
Mina R. Bionta $\odot,{ }^{1,}{ }^{*}$ Elissa Haddad, ${ }^{1}$ Adrien Leblanc, ${ }^{1}$ Vincent Gruson, ${ }^{1,2}$ Philippe Lassonde, ${ }^{1}$ Heide Ibrahim $\odot,{ }^{1}$ \\ Jérémie Chaillou, ${ }^{1}$ Nicolas Émond $\odot,{ }^{1}$ Martin R. Otto $\odot,{ }^{3}$ Álvaro Jiménez-Galán, ${ }^{4}$ Rui E. F. Silva, ${ }^{5}$ Misha Ivanov, ${ }^{4,6,7}$ \\ Bradley J. Siwick, ${ }^{3}$ Mohamed Chaker, ${ }^{1}$ and François Légaré ${ }^{1, \dagger}$ \\ ${ }^{1}$ Centre Énergie Matériaux et Télécommunications, Institut National de la Recherche Scientifique, 1650 Boulevard Lionel-Boulet, Varennes, \\ Québec, Canada J3X1S2 \\ ${ }^{2}$ Department of Physics, The Ohio State University, 191 West Woodruff Avenue, Columbus, Ohio 43210, USA \\ ${ }^{3}$ Department of Physics and Department of Chemistry, Center for the Physics of Materials, McGill University, 801 Sherbrooke Street W, \\ Montréal, Québec, Canada H3A OB8 \\ ${ }^{4}$ Max-Born-Institute, Max-Born Straße 2A, D-12489 Berlin, Germany \\ ${ }^{5}$ Departamento de Física Teórica de la Materia Condensada, Universidad Autónoma de Madrid, E-28049 Madrid, Spain \\ ${ }^{6}$ Department of Physics, Humboldt University, Newtonstraße 15, D-12489 Berlin, Germany \\ ${ }^{7}$ Department of Physics, Imperial College London, South Kensington Campus, SW7 2AZ London, United Kingdom
}

(Received 2 June 2020; revised 2 November 2020; accepted 3 June 2021; published 28 June 2021)

\begin{abstract}
We establish time-resolved high harmonic generation (tr-HHG) as a powerful spectroscopy method for tracking photoinduced dynamics in strongly correlated materials through a detailed investigation of the insulatorto-metal phase transitions in vanadium dioxide. We benchmark the technique by comparing our measurements to established momentum-resolved ultrafast electron diffraction, and theoretical density functional calculations. Tr-HHG allows distinguishing of individual dynamic channels, including a transition to a thermodynamically hidden phase. In addition, the HHG yield is shown to be modulated at a frequency characteristic of a coherent phonon of the equilibrium monoclinic phase over a wide range of excitation fluences. These results demonstrate that tr-HHG is capable of tracking complex dynamics in solids through its sensitivity to the band structure.
\end{abstract}

DOI: 10.1103/PhysRevResearch.3.023250

\section{INTRODUCTION}

As ultrashort light and electron sources have become more advanced, real-time dynamics of complex states of matter may be understood in greater detail [1,2]. High-harmonic generation (HHG) in atoms and molecules is a well-known process for generating table-top ultrafast sources from the extreme ultraviolet (EUV) to the soft x-ray spectral range, and has been used for probing dynamics in matter [1,3-5]. High-harmonic spectroscopy [3] has had great success probing electronic structure and dynamics in atoms, molecules [5-8], as well as in following chemical reactions in the gas phase [9-11]. Extending the generation of high-harmonics to solid state systems [12-14] allows for highly sensitive probing of the band structure of the generating material [15-19].

HHG was recently extended to solids, as first reported by Ghimire et al., who observed high harmonics generated from $\mathrm{ZnO}$ [12]. Over the years, two types of harmonics

\footnotetext{
*Present address: Research Laboratory of Electronics, Massachusetts Institute of Technology, 77 Massachusetts Avenue, Cambridge, Massachusetts, 02139, USA; mbionta@mit.edu

${ }^{\dagger}$ francois.legare@inrs.ca

Published by the American Physical Society under the terms of the Creative Commons Attribution 4.0 International license. Further distribution of this work must maintain attribution to the author(s) and the published article's title, journal citation, and DOI.
}

generated in solids have been identified: intraband and interband [13-15,18-22]. In both cases, the first step consists of multiphoton/tunneling excitation from a valence to a conduction band. For intraband harmonics, the generated radiation originates from a nonlinear band current driven by the laser field; for interband harmonics, the generation mechanism involves recombination of the accelerated electron and hole similar to HHG from atoms and molecules. In both cases, the harmonic spectra generated by solids should be a very sensitive probe of ultrafast phenomena that involve changes to the band structure near the Fermi level. Thus far, most HHG measurements in solids have been performed on static systems; however, pump-probe experiments on $\mathrm{ZnO}$ have revealed that the interband HHG yield is suppressed upon photodoping and remains quenched for several tens of picoseconds until the electrons return to the ground state [15]. The theory of HHG in solids is at an early stage, but undergoing rapid development, and suggests that several of the characteristic features of MottHubbard physics should impact directly the HHG yield from strongly correlated materials [23-26]. This picture of HHG suggests a sensitivity to a range of nonequilibrium phenomena occurring in strongly correlated materials, since many of these-including insulator-to-metal transitions-are associated with a redistribution of spectral weight or optical conductivity over several $\mathrm{eV}$, the energy scale on which $\mathrm{HHG}$ is most sensitive.

The current study takes an experimental approach to test this hypothesis by investigating how the time-resolved HHG yield (tr-HHG) changes during the photoinduced 
insulator-to-metal phase transitions in the strongly correlated material vanadium dioxide $\left(\mathrm{VO}_{2}\right)$. Strongly correlated materials are of particular interest because any optical excitation that modifies the interplay between lattice, charge, orbital, or spin degrees of freedom can result in dramatic transformations in their properties [27-33]. In practice, the optical perturbation is typically associated with the excitation of carriers (photodoping) or strongly driving a specific infrared-active lattice mode (phonon pumping). Generally, a complete picture of these transient, optically induced phenomena in materials requires the use of complementary techniques to reveal both the structure and properties of the nonequilibrium states, and the associated changes to the coupling between various degrees of freedom. Time-resolved techniques including $\mathrm{x}-$ ray or electron scattering [2,34], photoelectron, and transient spectroscopies ranging from the terahertz to the $\mathrm{X}$-ray regime have become the methods of choice for this burgeoning field $[35,36]$.

Photoexcited $\mathrm{VO}_{2}$ exhibits a rich phenomenology enabled by its multiband Mott-Hubbard character [35-49]. Recently, it has been shown that there are two qualitatively distinct photoinduced insulator-to-metal phase transitions (IMT) in $\mathrm{VO}_{2}$ following photoexcitation [35-37,50]. The first one, accessible at relatively high pump fluence, is an analog of the equilibrium phase transition, and is associated with the lattice-structural transition between the monoclinic insulator $\left(M_{1}\right)$ and the rutile metallic $(R)$ crystallography expected from the equilibrium phase diagram [39,41-44,46,48,48,49]. The second one, accessible at lower pump fluence, has no equilibrium/thermodynamic analog and yields a metastable, monoclinic metal phase $(\mathcal{M})$ that retains the crystallographic symmetry of its parent equilibrium monoclinic phase, but exhibits a novel one-dimensional (1D) antiferroelectric charge order not present at equilibrium [36]. Furthermore, this hidden phase transition of primarily electronic character has also been identified through numerical simulations using density functional theory [37].

In Fig. 1, schematic partial density of states diagrams for these three phases $\left(M_{1}, \mathcal{M}\right.$, and $\left.R\right)$ are presented. There is broad agreement that the principal changes in band structure associated with these transitions occur in the bands formed by the $\mathrm{V}_{3 d}$ states of $t_{2 g}$ symmetry $\left(d_{x 2 y 2} \rightarrow d_{\|}, d_{x y} \rightarrow\right.$ $d_{\pi}^{\perp}, d_{x z} \rightarrow d_{\pi}^{\|}$) [51,52]. In the $M_{1}$ phase, the optical band gap is formed between the $d_{\|}$and $d_{\pi *}^{\|}$bands $[40,41,49]$. In the $R$ phase, the band gap collapses and the $d$ bands are effectively degenerate at the Fermi level $[40,41,49]$. In the $\mathcal{M}$ phase, recent calculations suggest a reordering of $d_{\|}$and $d_{\pi *}$ bands yielding a partial overlap at the Fermi level [37].

We show that tr-HHG reports on each of these previously demonstrated features in all three phases of $\mathrm{VO}_{2}$, benchmarking the technique as an all-optical method for probing dynamics in strongly correlated materials.

\section{RESULTS}

In these experiments, a mid-infrared (MIR) laser pulse called the driver, centered at either 10 or $7 \mu \mathrm{m}$, with a pulse duration of $80 \mathrm{fs}$ and peak intensity in the range of $\sim 2 \times 10^{12}$ $\mathrm{W} \mathrm{cm}{ }^{-2}$, is used to drive $\mathrm{HHG}$ from a $100 \mathrm{~nm}$ thick, epitaxial $\mathrm{VO}_{2}$ sample [38] as shown in Fig. 2(a). The $\mathrm{VO}_{2}$ sample, in (a)
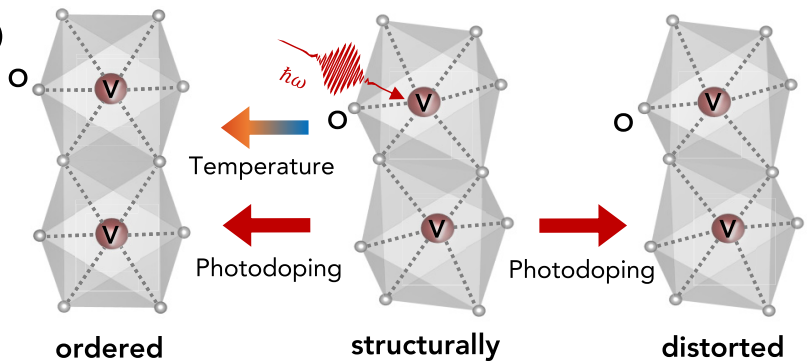

ordered

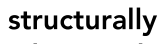

distorted

distorted charge-order?

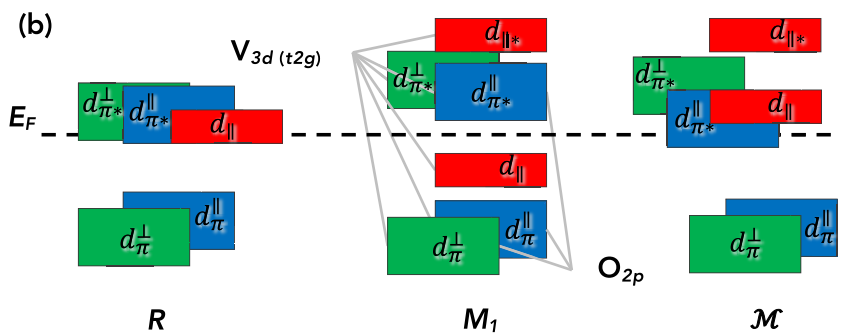

FIG. 1. Atomic and band structure of the $\mathrm{VO}_{2}$ IMT. (a) Atomic arrangement of $\mathrm{VO}_{2}$ during IMT phase transitions. In the photoinduced phase transition, the $M_{1} \rightarrow R$ transition occurs at high fluence, while the $M_{1} \rightarrow \mathcal{M}$ occurs at low to moderate fluences. (b) Energy diagram of the band structure of $\mathrm{VO}_{2}$ in the $R, M_{1}$, and $\mathcal{M}$ phases.

the $M_{1}$ phase at a room temperature of $20^{\circ} \mathrm{C}$, is photoexcited with a 50 fs laser pulse centered at $1.5 \mu \mathrm{m}$, called the pump, to initiate the IMT. The generation of the fifth harmonic of the $10 \mu \mathrm{m}$ HHG driver (at $2.0 \mu \mathrm{m}$ ) or third harmonic of the $7 \mu \mathrm{m}$ driver (at $2.3 \mu \mathrm{m}$ ) is measured as a function of time delay between the pump and driver for various pump fluences by a spectrometer or photodiode, as presented in Fig. 2(a). Given the driver intensity and wavelength, and that the measured harmonic is below the band gap of $\mathrm{VO}_{2}(0.68 \mathrm{eV}$, $1.815 \mu \mathrm{m}$ ), the HHG signal is expected to be dominated by intraband harmonics $[18,21]$. This is further confirmed by numerical simulations of a semiconductor with the same band gap as $\mathrm{VO}_{2}$ (see Appendix D). These measurements are taken in both the forward and backward directions (for backward measurements, see Appendix F). Furthermore, pump-probe transmissivity measurements are performed with 50 fs infrared (IR) probe pulses at $1.7 \mu \mathrm{m}$. More details about the experimental geometry can be found in Appendix A.

In Fig. 2(b), we present HHG spectra for two temperatures, $293 \mathrm{~K}\left(20{ }^{\circ} \mathrm{C}\right)$ and $373 \mathrm{~K}\left(100{ }^{\circ} \mathrm{C}\right)$ driven by 10 $\mu \mathrm{m}$. When heated above $\sim 343 \mathrm{~K}\left(70{ }^{\circ} \mathrm{C}\right)$ [39], $\mathrm{VO}_{2}$ undergoes an insulator-to-metal transition that is associated with a change in crystallography from the monoclinic insulator $\left(M_{1}\right)$ to metallic rutile $(R)$ phase. In the $M_{1}$ phase we observe up to the seventh harmonic in the HHG spectra, while for the $R$ phase there is significant suppression of the harmonic yield with no measurable harmonics [Fig. 2(b)]. This is consistent with the collapse of the band gap in $R$ phase $\mathrm{VO}_{2}$ [41,51,52] [Fig 1(b)], decreasing the anharmonicity of the bands required for HHG and suppressing the generation of harmonics. The same behavior is observed in the backward direction (see Appendices B and F). We note that the $R$ phase is metallic. From a microscopic 
(a)
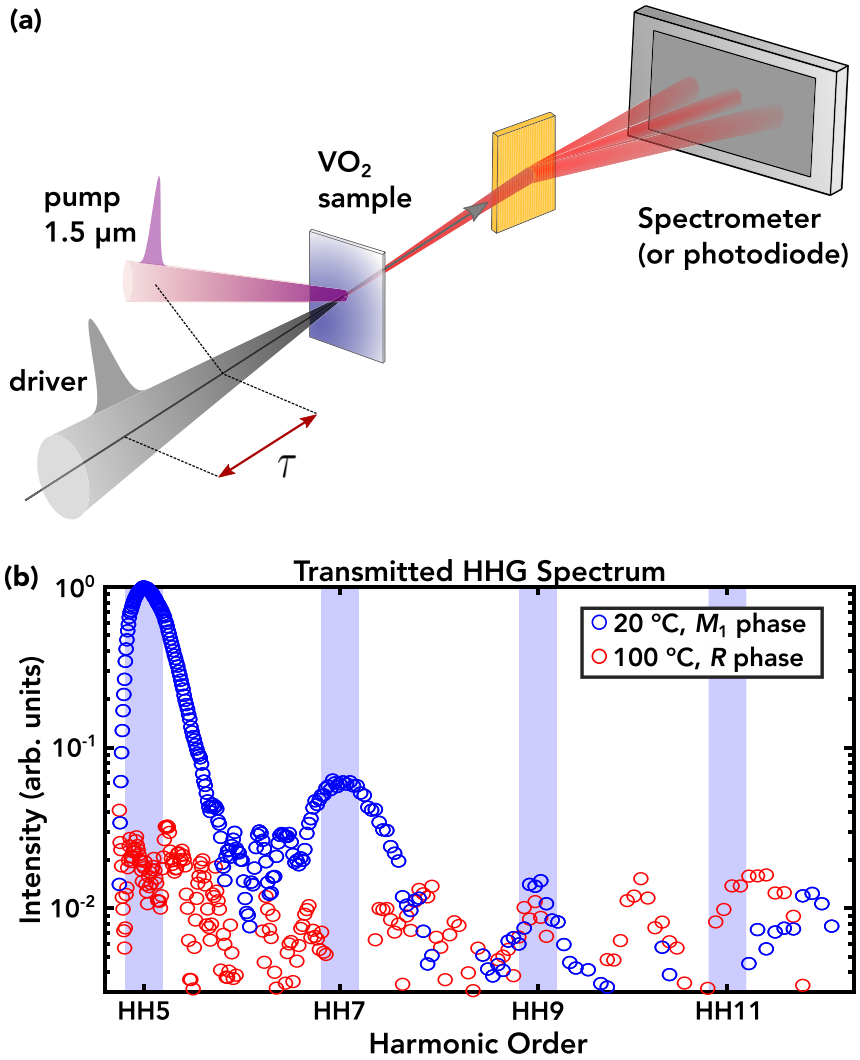

FIG. 2. Experimental geometry. (a) An $80 \mathrm{fs}$, MIR driver pulse is used to generate harmonics from a $100 \mathrm{~nm}$ thick epitaxial $\mathrm{VO}_{2}$ sample. The transmitted harmonic spectrum is then collected and recorded using a spectrometer or photodiode. A $50 \mathrm{fs}, 1.5 \mu \mathrm{m}$ pump at variable delay, $\tau$, from the driver is used to photoexcite the IMT. The fluence of the pump can be modulated using a half-waveplate, polarizer energy throttle. (b) The unpumped high harmonic (HH) spectrum driven by $10 \mu \mathrm{m}$ in the $M_{1}$ phase at room temperature of $20{ }^{\circ} \mathrm{C}$ (blue circles) and $R$ phase at $100{ }^{\circ} \mathrm{C}$ (red circles). Blue rectangles indicate locations of expected harmonics.

perspective, electrons in the metallic phase behave as nearly free particles, and therefore their response will be essentially linear, suppressing the generation of high harmonics. Also, the stronger dephasing in the metal will further reduce the harmonic yield. From a macroscopic viewpoint, the laser penetration depth in a metal is extremely small. Thus, we expect a significant drop of the harmonic yield in the time-resolved experiments for the photoinduced IMT.

Before we present the tr-HHG measurements, we report the pump-probe transmissivity measurements. In Figs. 3(a) and 3 (b), at pump fluences greater than $6 \mathrm{~mJ} \mathrm{~cm}^{-2}$, we observe a drop in the optical transmissivity at $1.7 \mu \mathrm{m}$, whose magnitude monotonically increases with the pump fluence. The magnitude of the amplitude drop with respect to pump fluence is shown as pink triangles in Fig. 4(d), clearly demonstrating a monotonic behavior. This initial drop, limited by the pump pulse duration $[38,40,41]$, is followed by a flat response within the temporal window of $10 \mathrm{ps}$. Within this timescale, there is no recovery of the IR transmissivity. Similar measurements have been performed with a $1.3 \mu \mathrm{m}$ pump and $4 \mu \mathrm{m}$ probe showing these same dynamics with no recovery in transmissivity observed (Appendix G).
Figures 3(c) and 3(d) show the tr-HHG measurements for the fifth harmonic driven by $10 \mu \mathrm{m}$. At high pump fluence, the measurements are qualitatively similar to the transmissivity measurements at $1.7 \mu \mathrm{m}$. Pump fluences greater than $35 \mathrm{~mJ} \mathrm{~cm}^{-2}$ lead to a nearly complete suppression of the harmonic yield at time zero with no recovery observed over 10 ps [see Fig. 3(c)]. These dynamics are very similar to recent tr-HHG measurements in the semiconductor $\mathrm{ZnO}$, where the HHG yield is greatly suppressed by photodoping, recovering only after several tens of picoseconds once the system returns to the ground electronic state [15]. Recent UED measurements $[35,36]$ show that - at these pump fluences-approximately $80 \%$ of the film undergoes the photoinduced $M_{1}$ to $R$ phase transition. Thus, the significant suppression of $\mathrm{HHG}$ for $\mathrm{VO}_{2}$ for high pump fluences where the material undergoes a transition to the $R$ phase is consistent with the temperature dependent HHG measurements presented previously [see Fig. 2(b)].

If the pump fluence is very low, $\lesssim 3 \mathrm{~mJ} \mathrm{~cm}^{-2}$, both the IR transmissivity and harmonic yield experience a small suppression at time zero that rapidly recovers (within $500 \mathrm{fs}$ ) to very nearly the same signal levels before photoexcitation. This indicates that at low pump fluence no phase transitions are induced, and $\mathrm{VO}_{2}$ rapidly returns to the equilibrium $M_{1}$ phase [Figs. 3(c) and 3(d)]. This observation is also in good agreement with UED measurements and several other timeresolved spectroscopic measurements $[35,36,38,41]$.

In the fluence range from 3 to $35 \mathrm{~mJ} \mathrm{~cm}^{-2}$, however, there is a striking difference between the time-resolved transmissivity and the tr-HHG measurements. Immediately after photoexcitation, both signals exhibit a drop, limited in time by the pump pulse duration, whose amplitude monotonically increases with pump fluence. The tr-HHG yield from $\mathrm{VO}_{2}$ then demonstrates a recovery on the picosecond timescale, much faster than the recovery of its own IR transmissivity. This recovery of the tr-HHG yield is characterized by a biexponential with an amplitude that is non-monotonic with pump fluence. This recovery has two timescales: a fast one of $\sim 300$ fs followed by a second slow one of $\sim 1.5$ ps [see Fig. 4(c) and Appendix C]. The fast term, here called the $M_{1}^{*} \rightarrow M_{1}^{*, b}$ transition, shows reasonable agreement with the recent numerical calculations describing thermalization of the photodoped carrier distributions [37]. The slow term matches UED measurements of the $\mathcal{M}$ phase formation time [35,36]. The behavior is also observed in the backward directed tr-HHG (Appendix F) as well as in the tr-HHG dynamics of the third harmonic driven by $7 \mu \mathrm{m}$ pulses [Figs. 4(a) and 4(b)). This is in striking contrast with the tr-HHG measurements on $\mathrm{ZnO}$, where no recovery of the interband harmonics were observed [15]. For our simulations of a simple semiconductor with the same band gap as $\mathrm{VO}_{2}$, we see an increase in harmonic yield due to the injection of photodoped carriers (see Appendix D). This increase in yield was also predicted by Wang et al. [15] for intraband harmonics and is opposite of what was observed experimentally.

\section{DISCUSSION}

From UED measurements [35,36], it is known that the polycrystalline $\mathrm{VO}_{2}$ sample demonstrates a multi-phase heterogeneous response over this range of pump fluences 

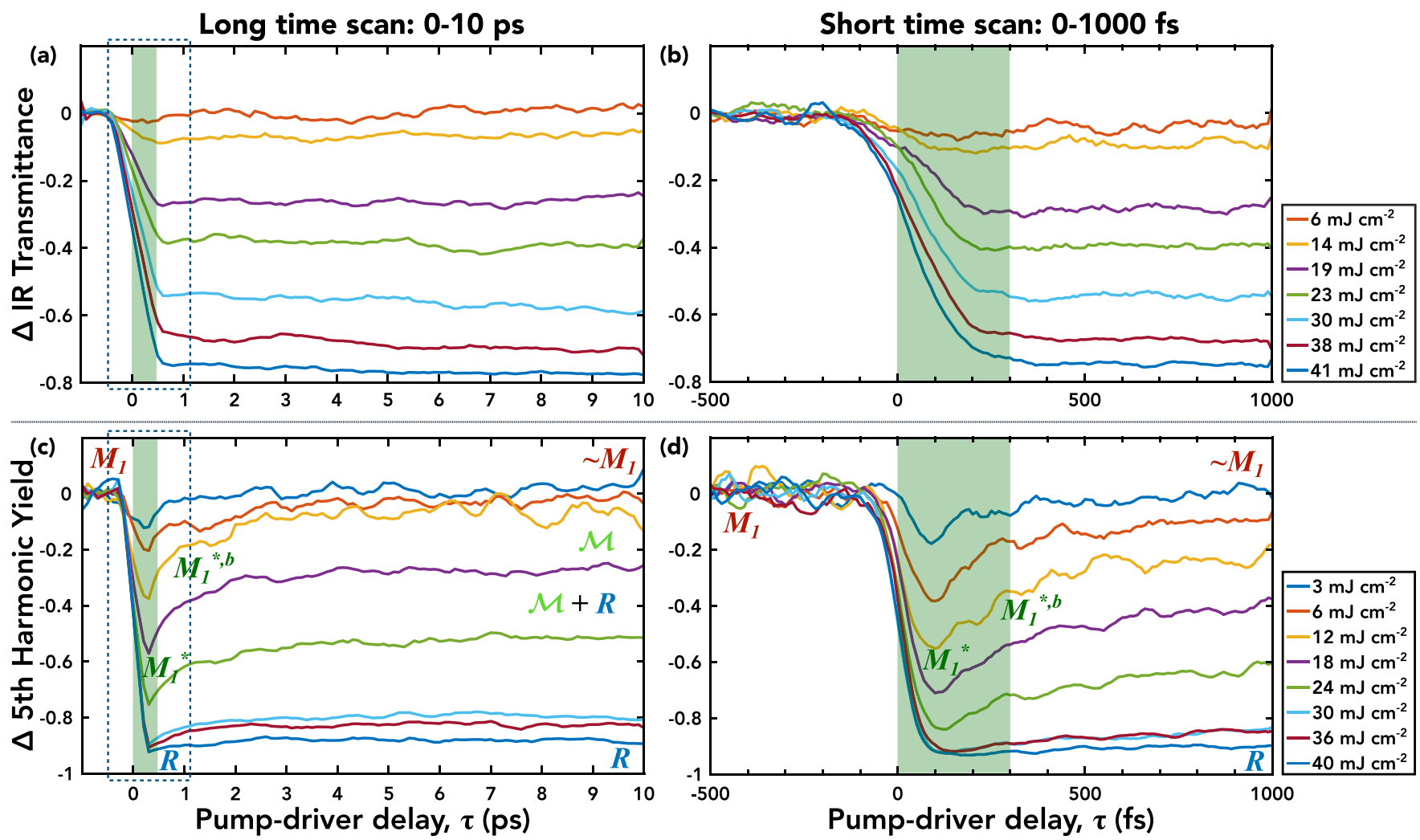

FIG. 3. Change in optical IR transmittance (top) and fifth harmonic yield (bottom). The time dependent change in optical IR transmittance (top) or the fifth harmonic yield (bottom) driven by $10 \mu \mathrm{m}$ following the photoexcited IMT in $\mathrm{VO}_{2}$. Negative delays indicate the harmonic generating driver or IR probe pulse arrives before photoexcitation. The various phases of $\mathrm{VO}_{2}$ are annotated on the curves. No revival of the IR transmittance is observed for long timescales in (a) or short timescales in (b). (c) Long-term recovery and revival of the harmonics as the $\mathrm{VO}_{2}$ transitions to the $\mathcal{M}$ phase. The amplitude of $\mathcal{M}$ recovery in the HHG yield is plotted as a function of pump fluence in Fig. 4(d). The dashed rectangles are expanded upon with higher time-resolution in (b) and (d). The shaded green rectangles show the region of the fast dynamics for the $M_{1}^{*} \rightarrow M_{1}^{*, b}$ transition. In both (c) and (d) the suppression of the harmonics in the $R$ phase can be seen for high fluences.

(3-35 $\mathrm{mJ} \mathrm{cm}^{-2}$ ) due to the concurrence of the $M_{1} \rightarrow R$ and $M_{1} \rightarrow \mathcal{M}$ transitions. UED measurements showed that the phase fraction of the $\mathcal{M}$ phase increases up to a pump fluence of $\sim 20 \mathrm{~mJ} \mathrm{~cm}{ }^{-2}$ and then decreases at higher pump fluence where the rutile phase dominates. This is precisely the nonmonotonic behavior of the HHG signal recovery observed by the tr-HHG measurements. The amplitude of the HHG yield recovery is shown in Fig. 4(d) (red squares and diamonds) alongside the phase fraction of the $\mathcal{M}$ phase obtained by UED in [36]. The HHG yield recovery is given by the difference of the average change in harmonic yield for long delays ( $\tau=$ $8-10 \mathrm{ps}$ ) and the minimum change in harmonic yield observed right after $t_{0}$ normalized by the maximum change in harmonic yield from the $R$ phase from $\tau=8-10 \mathrm{ps}$ (pump fluence of 40 $\mathrm{mJ} \mathrm{cm}{ }^{-2}$ ). Note the similarity in shape between the amplitude of HHG recovery and the UED $\mathcal{M}$ phase fraction despite the UED samples being pumped with $800 \mathrm{~nm}$ compared to 1.5 $\mu \mathrm{m}$ as presented here. The decreasing monotonic behavior of the IR transmissivity is shown alongside the tr-HHG recovery in Fig. 4(d) as pink triangles.

The tr-HHG results from both driver wavelengths show a nonmonotonic recovery in the HHG yield through the pump fluence range studied compared with the IR optical transmissivity, which rapidly decreases and then remains always flat over 10 ps, with no recovery observed. Curiously, the HHG yield from the $\mathcal{M}$ phase appears to be very similar to that of the $M_{1}$ phase, despite being metallic with a low frequency $\mathrm{THz}$ average conductivity $\sim 1 / 3$ that of the equilibrium metallic $R$ phase [36]. This observation seems to be at odds with those shown in Fig. 2(b), where the $R$ phase is shown to have negligible HHG yield. The high production of harmonics in the $\mathcal{M}$ phase (see Fig. 3) indicates that despite this metallic character, as highlighted by a drop of transmissivity that persists for tens of picoseconds [see Fig. 3(a)], the bands near the Fermi level [see Fig. 1(b)] must maintain the electronic structure required for HHG [13-15,18-22]. This is a salient observation that does not follow from the earlier studies using more conventional optical spectroscopies, nor was it predicted by theory. These observations may be due to the strong correlations in the material and a hopping-like character to the conductivity, or a different sensitivity of optical and HHG yield to electron localization/delocalization processes and shifts in electronic band structure. However, the most likely explanation seems to be that the $\mathcal{M}$ phase is best described as 1D metal with high conductivity limited to a single crystallographic direction [53]. This direction is most likely to be the monoclinic $a$ axis (equivalent to the rutile $c$ axis), along which the vanadium atoms are dimerized, and 

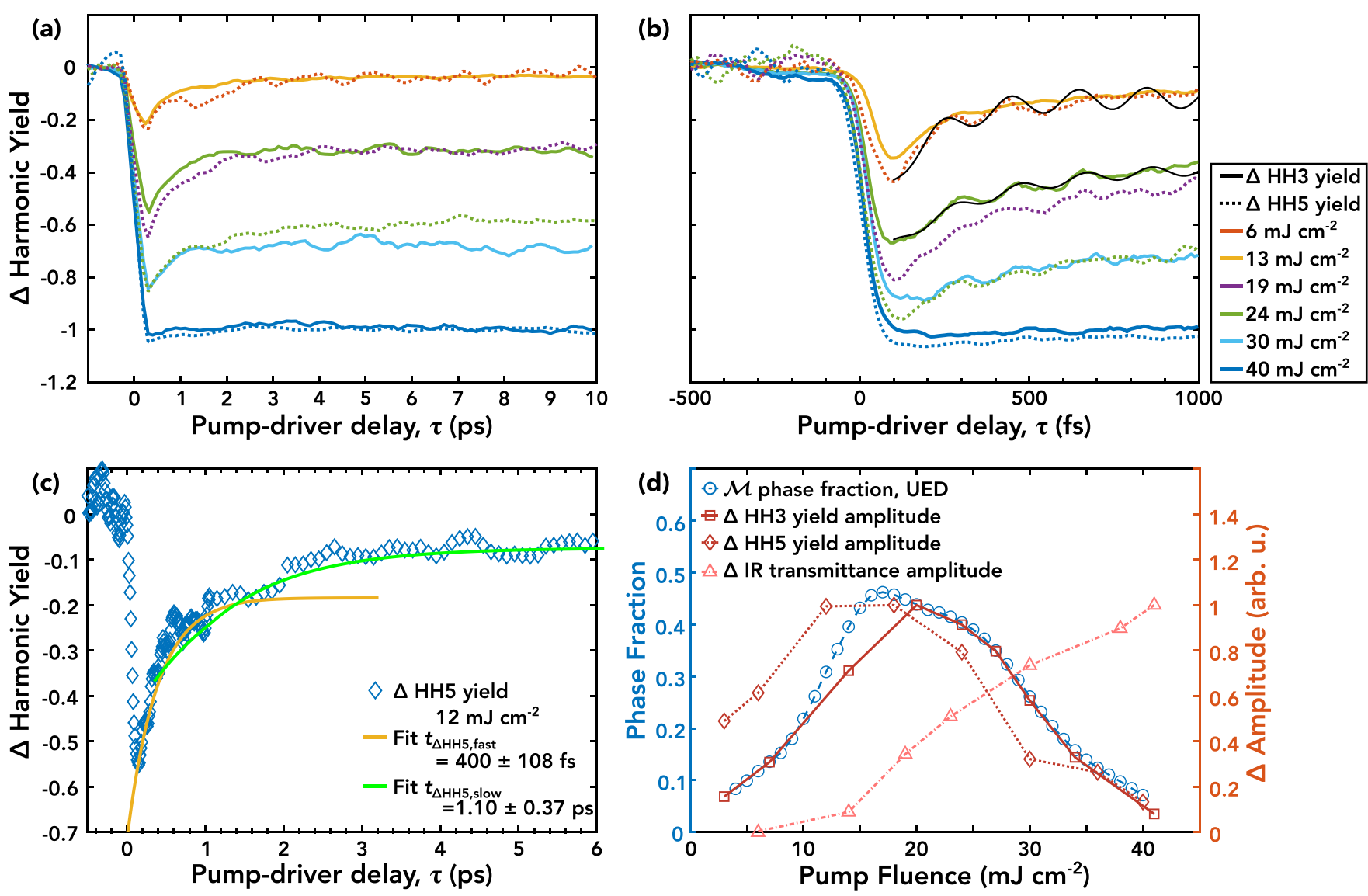

FIG. 4. HHG, IR transmittance, and UED comparisons. (a) and (b) Comparison of the fifth harmonic (solid lines, driven by $10 \mu \mathrm{m}$ ) and third harmonic (dashed lines, driven by $7 \mu \mathrm{m}$ ) dynamics for varying pump fluences. Similar dynamics are observed regardless of harmonic probed. Black oscillations indicated in (b) correspond to $M_{1}$ phase phonon modes (Appendix E). (c) Biexponential fit of a moderately pumped HHG yield where both the $M_{1}^{*} \rightarrow M_{1}^{*, b}$ and $M_{1}^{*, b} \rightarrow \mathcal{M}$ transitions are occurring (Appendix C). (d) Extracted phase fractions for the $\mathcal{M}$ phase from UED results [35,36] for different pump fluences, and the corresponding change in amplitude for the IR transmittance and HHG yield. Note the UED results were pumped with $800 \mathrm{~nm}$, and the tr-HHG was pumped with $1.5 \mu \mathrm{m}$.

antiferroelectric charge ordering in the $\mathcal{M}$ phase has been observed in UED experiments [36]. If the $\mathcal{M}$ phase is a 1D metal, it could simultaneously present metallic conductivity when probed by tr-THz spectroscopy [36] and maintain a high HHG yield as observed in the current measurements. This suggests that the picture presented in Fig. 1(b) for the $\mathcal{M}$ phase should be modified to include directions perpendicular and parallel to the monoclinic $a$ axis. Along the $a$ axis, there is band overlap similar to $R$, but perpendicular to the $a$ axis an anharmonic band gap remains, thus fulfilling the conditions for HHG.

We should reiterate that the phase transition to the monoclinic metal phase $(\mathcal{M})$ is independent of the $M_{1} \rightarrow R$, appearing as a parallel transformation channel that is open across a wide range of pump fluences. The $\mathcal{M}$ state is not an intermediary step along the $M_{1} \rightarrow R$ transition pathway as was incorrectly implied in Ref. [42] (see note in Ref. [36]). Here we provide a independent observations of the $\mathcal{M}$ phase, using an all-optical technique. Furthermore, there have been other independent measurements of this $\mathcal{M}$ phase as well [50].

To help further clarify the tr-HHG data interpretation and highlight the salient features of the data presented here, we have performed calculations of the tr-HHG yield for a conventional band-semiconductor model following photoexcitation (Appendix D). These calculations demonstrate that photoexcitation above the band gap (i.e., photodoping electrons and holes) is expected to increase HHG yield in a semiconductor that does not exhibit a substantial band gap renormalization. This is precisely the opposite of the HHG yield dynamics observed in $\mathrm{VO}_{2}$ following photoexcitation. Thus, the decrease in $\mathrm{HHG}$ yield in $\mathrm{VO}_{2}$ cannot be understood as a direct photocarrier effect. The changes in yield observed is evidence of band gap dynamics in $\mathrm{VO}_{2}$ : a photoinduced collapse of the band gap followed by partial recovery on the timescale of $\mathcal{M}$ formation over a range of fluences. A more sophisticated modeling of the $\mathrm{HHG}$ yield that includes the correlated electron behavior of $\mathrm{VO}_{2}$ is currently a major theoretical challenge and out of the scope of this paper (see Appendix D).

Another notable feature of the tr-HHG yield is the presence of coherent oscillations during the recovery [see the black curves in Fig. 4(b)], with timescales matching coherent phonon dynamics. These phonon modes, alongside photodoped electrons, provide a pathway for the excess energy from the photoexcitation to reach equilibrium. Raman spectroscopy studies on both the insulating monoclinic 
phase and rutile metallic phase show distinct active phonon modes: 18 in the monoclinic phase and 4 in the rutile phase $[40,47,48,54]$. Despite the low signal-to-noise ratio of our measurements due to the $50 \mathrm{~Hz}$ repetition rate of our laser system, we are able to observe oscillations for low to moderate pump fluences up to $30 \mathrm{~mJ} \mathrm{~cm} \mathrm{~cm}^{-2}$ for both the fifth harmonic of $10 \mu \mathrm{m}$ and third harmonic of $7 \mu \mathrm{m}$. They are found to have a frequency on the order of $6 \pm 2 \mathrm{THz}$ encompassing the lowest two order phonon modes known for monoclinic $\mathrm{VO}_{2}$ (at 4.4 and $5.7 \mathrm{THz}$ ). The persistence of these phonon dynamics remains for pump fluences much higher than those reported in $[40,47,48]$ via time-resolved reflectivity measurements and suggest that the tr-HHG technique can map the coherent phonon dynamics [55]. The origin of this modulation can be either a change in band gap, thus modulating the photoexcitation probability, or the anharmonicity of the band. At present, our measurements cannot identify the specific mechanism responding to the phonon modes which leads to the modulation of the tr-HHG yield. Yet our measurements of coherent phonon dynamics via tr-HHG is similar to observations of nuclear dynamics with HHG spectroscopy of photoexcited gas phase molecules $[10,11]$. The presence of these phonon modes for the monoclinic phase $\mathrm{VO}_{2}$ and not the rutile phase indicates that although there is an initial drop in harmonic yield for the photoexcited state, the material retains monoclinic symmetry, and has not yet transitioned to the rutile phase. In the future, it should be possible to significantly improve the signal-to-noise ratio of these measurements, using mid-IR optical parametric chirpedpulse amplification (OPCPA) running at $\mathrm{kHz}$ repetition rates [56] to further investigate the observed modulations. Further details on the oscillations and their analysis can be found in Appendix E.

\section{CONCLUSION}

We have demonstrated tr-HHG spectroscopy from a strongly correlated material. We have shown that tr-HHG is capable of discriminating and tracking the real-time evolution of the distinct phase transitions and associated coherent phonon dynamics in $\mathrm{VO}_{2}$, including observations of the hidden metastable $\mathcal{M}$ phase that has no equilibrium analog. This technique provides complementary information to UED and optical measurements of similar nature. As tr-HHG spectroscopy is highly sensitive to the band structure of a material, it can be used to probe phase transitions in materials as their band structures change. For example, we observe that although the metastable $\mathcal{M}$ phase in $\mathrm{VO}_{2}$ presents with metallic optical properties, the band structure in this phase allows for a high HHG yield that is directly correlated to the $\mathcal{M}$ phase fraction. Due to the sensitivity of tr-HHG, we expect that tr-HHG spectroscopy can be extended to other strongly correlated materials and other solids, to detect some of the promising features listed in [27] and to gain information about the dynamics as the systems evolve through different electronic states. With a simple experimental setup to implement, the tr-HHG spectroscopy technique developed in this work opens the path to the study of how materials evolve and transform to exotic phases under such different conditions as high pressure, high temperature, and photoexcitation. Moreover, with this setup, we can vary the angle of polarization or incidence, paving the way to retrieve parallel information between tr-HHG and other angle resolved spectroscopy measurements for the study of $2 \mathrm{D}$ materials.

\section{ACKNOWLEDGMENTS}

We thank Antoine Laramée for his technical contributions. We thank Andrew Bruhács and Luke Govia for their scientific discussions. We acknowledge funding from NSERC, FRQNT, MEI, and CFI-MSI. V.G. was supported by the Air Force Office of Science Research under MURI Award No. FA955016-1-0013. M.I. and Á.J.G. acknowledge support from the European Union's Horizon 2020 research and innovation program under Grant Agreement No. 899794.

M.R.B and F.L conceived the experiments. M.R.B. performed the experiments with assistance from E.H., V.G., A.L., H.I., and P.L. J.C., N.É., and M.C. fabricated and characterized the samples. Á.J.G., R.E.F.S., and M.I. performed the theoretical calculations. M.R.B. analyzed the results with support from H.I. and F.L. M.R.B. wrote the manuscript and supplementary information with significant contributions from V.G., A.L., H.I., M.R.O., B.J.S., and F.L. All authors contributed to revising and editing the manuscript.

\section{APPENDIX A: METHODS}

\section{Experimental setup}

These experiments were performed on the $50 \mathrm{~Hz}$ beamline at the Advanced Laser Light Source user facility (ALLS, Varennes, QC, Canada). A high energy optical parametric amplifier (HE-OPA) was used to generate the mid-infrared (MIR) source used to drive high harmonic generation (HHG) in $\mathrm{VO}_{2}$. This OPA line is pumped by $1.1 \mathrm{~mJ}$ of $800 \mathrm{~nm}$, Ti:sapphire laser light at a $50 \mathrm{~Hz}$ repetition rate, producing $150 \mu \mathrm{J}$ of $1.7 \mu \mathrm{m}$ pulses using a commercial two-stage TOPAS system (Light Conversion). The output is further amplified in a $20 \times$ $20 \times 1 \mathrm{~mm}$, Type-I BBO crystal $\left(\theta=19.9^{\circ}\right)$ using $20 \mathrm{~mJ}$ of $800 \mathrm{~nm}$ light. From this, $7 \mathrm{~mJ}$ of signal $(1.5 \mu \mathrm{m})$ and idler $(1.7 \mu \mathrm{m})$ is produced that is then used to drive the difference frequency generation (DFG) stage. A $10 \mu \mathrm{m}$ MIR source is obtained by DFG in a $100 \mu \mathrm{m}$ thick GaSe crystal at $30^{\circ}$ to produce $\sim 20 \mu \mathrm{J}$ of pulse energy. The MIR was characterized using a Spectral Products grating-based slit monochromator, with a liquid-nitrogen-cooled $\mathrm{HgCdTe}$ detector, with a central wavelength found to be $10 \mu \mathrm{m}$, and with a pulse duration of $80 \mathrm{fs}$ full width at half maximum (fwhm), measured via frequency resolved optical switching [57]. The OPA line can also be tuned such that the DFG generates $7 \mu \mathrm{m}$ pulses with pulse duration and energy similar to the $10 \mu \mathrm{m}$ pulses.

A surface reflection from a thin wedge is taken from the signal arm of the DFG setup to pump the phase transitions in $\mathrm{VO}_{2}$ at a wavelength of $1.5 \mu \mathrm{m}$. The fluence of this pump is modulated using a half-waveplate, Ge polarizer energy throttle and slightly focused using a $150 \mathrm{~mm}$ lens to achieve a fluence on the order of tens of $\mathrm{mJ} \mathrm{cm}^{-2}$. The delay between the $1.5 \mu \mathrm{m}$ pump and the MIR driver is varied using a Thorlabs $2825 \mathrm{~B}$ dc stepper motor actuator. The pulse duration of the pump was characterized using a home-built 
second harmonic generation frequency resolved optical gating (SHG-FROG) setup and found to be $50 \mathrm{fs}$ fwhm.

$13 \mu \mathrm{J}$ of MIR is focused onto the $\mathrm{VO}_{2}$ sample with an off-axis parabola (OAP) with a reflected focal length of $40 \mathrm{~mm}$. This gives us an intensity of $\sim 1.7 \times 10^{12} \mathrm{~W} \mathrm{~cm}^{-2}$, and a Keldysh parameter much less than 1, thus we are in the tunneling regime [58,59]. The $\mathrm{HHG}$ yield from the $\mathrm{VO}_{2}$ driven by $10 \mu \mathrm{m}$ is collected using an Ocean Optics NIR256 spectrometer starting from the fifth harmonic. Tr-HHG measurements cannot be performed using the seventh harmonic as it is too close to the pump wavelength to be spectrally separated. The third harmonic driven by $7 \mu \mathrm{m}$ is collected using a Thorlabs DET10D2 biased InGaAs detector with a long pass filter at $1700 \mathrm{~nm}$. Once again, the tr-HHG measurements from the fifth harmonic cannot be separated from the pump wavelength. The HHG spectra were taken using the spectrometer and the amplitude of the fifth harmonic yield was measured using the photodiode. Unless otherwise specified, the experiments were performed in ambient conditions at a room temperature of $20{ }^{\circ} \mathrm{C}$. HHG measurements were taken in both transmission and in the backward direction. Complementary pump-probe transmissivity measurements were performed using the $50 \mathrm{fs}, 1.7 \mu \mathrm{m}$ idler pulses as the probe.

\section{Sample preparation}

The epitaxial $\mathrm{VO}_{2}$ samples were deposited onto a $532 \mu \mathrm{m}$ $r$-cut sapphire substrate via reactive pulsed laser deposition (PLD) and grown to a thickness of $100 \mathrm{~nm}$ at the Laboratory of Micro and Nanofabrication (LMN, Varennes, QC, Canada). The measured band gap $\left(E_{g}\right)$ of the sample is found to be $E_{g}=0.683 \pm 0.002 \mathrm{eV}(1815 \mathrm{~nm})$. Details of the deposition process and characterization of the sample can be found in Bionta et al. [38].

\section{APPENDIX B: $\boldsymbol{R}$ PHASE MEASUREMENTS}

For comparison to the photoexcited phase transition with high fluence to the $R$ phase, measurements were performed at a temperature of $100{ }^{\circ} \mathrm{C}$. This temperature is well above the critical temperature for the IMT in $\mathrm{VO}_{2}$ of $\sim 343 \mathrm{~K}\left(\sim 70{ }^{\circ} \mathrm{C}\right)$ so the sample is sure to be in the $R$ phase. The generated high harmonic spectra in the forward and backward [60] direction are compared for the $M_{1}$ vs $R$ phases in Fig. 5 .

\section{APPENDIX C: FITTING}

From the UED and time resolved (tr-)HHG results, timescales for all three phase transitions can be found.

\section{UED results from Morrison et al. [35]}

Time constants for the $M_{1} \rightarrow R$ and $M_{1} \rightarrow \mathcal{M}$ phase transitions were found from the UED results of Morrison et al. [35]. We compared the time constant found for the $M_{1} \rightarrow \mathcal{M}$ phase transition with that obtained from the tr-HHG.

$M_{1} \rightarrow \mathcal{M}$ phase transition. The $M_{1} \rightarrow \mathcal{M}$ time constant was found by fitting the UED results of Morrison et al. [35] for formation of the $\mathcal{M}$ phase to a time constant of $t_{\mathrm{UED} \text {, slow }}=$ $1.6 \pm 0.2 \mathrm{ps}$ to the changing (200) and (220) peaks that are present for both the monoclinic and rutile phases in $\mathrm{VO}_{2}$
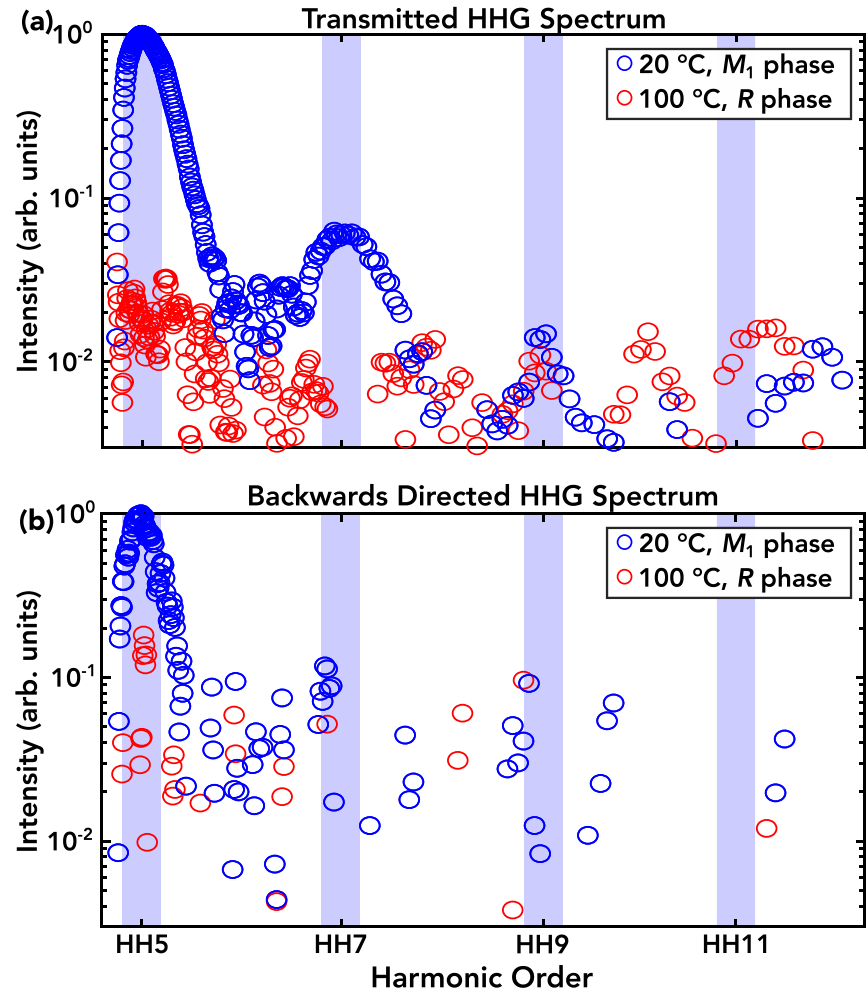

FIG. 5. $M_{1}$ vs $R$ phase harmonic generation. High harmonic spectra in the forward (top) and backward (bottom) direction for the $M_{1}$ phase (blue) at $20{ }^{\circ} \mathrm{C}$ and $R$ phase (red) at $100{ }^{\circ} \mathrm{C}$. Blue shaded rectangles show location of expected harmonics. $\mathrm{HH}$ : high harmonic.

(Fig. 6, red circles). If an $M_{1} \rightarrow R$ transition is initiated via photoexcitation, a $\sim 300 \mathrm{fs}$ time constant would be observed for the $(30 \overline{2})$ peak. However, a slower process is observed, even at lower pump fluences. This is because the atomic form factor term in the scattering intensity increases due to an electronic reorganization of the electrostatic potential since the diffracted intensity is sensitive to the valence charge distribution. This is attributed to the formation of the $\mathcal{M}$ state. Since the (200) and (220) are relatively low index peaks with a low scattering vector, they are sensitive to long range order in the sample. A time constant for the $M_{1} \rightarrow \mathcal{M}$ is found to be $t_{\text {slow }}=1.6 \pm 0.2$ ps (Fig. 6, red dashed line) when pumped with $20 \mathrm{~mJ} \mathrm{~cm}^{-2}$ at $800 \mathrm{~nm}$.

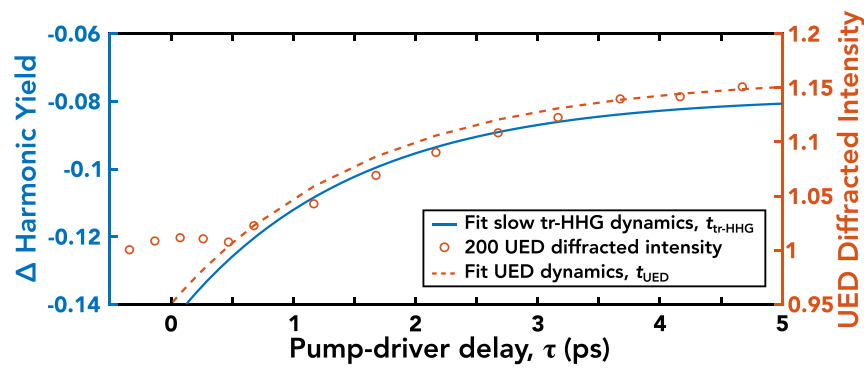

FIG. 6. Slow timescale. The slow timescale retrieved from third harmonic tr-HHG (blue curve) is in good agreement with results from UED measurements (data: red circles; fit: red dashed curve). 

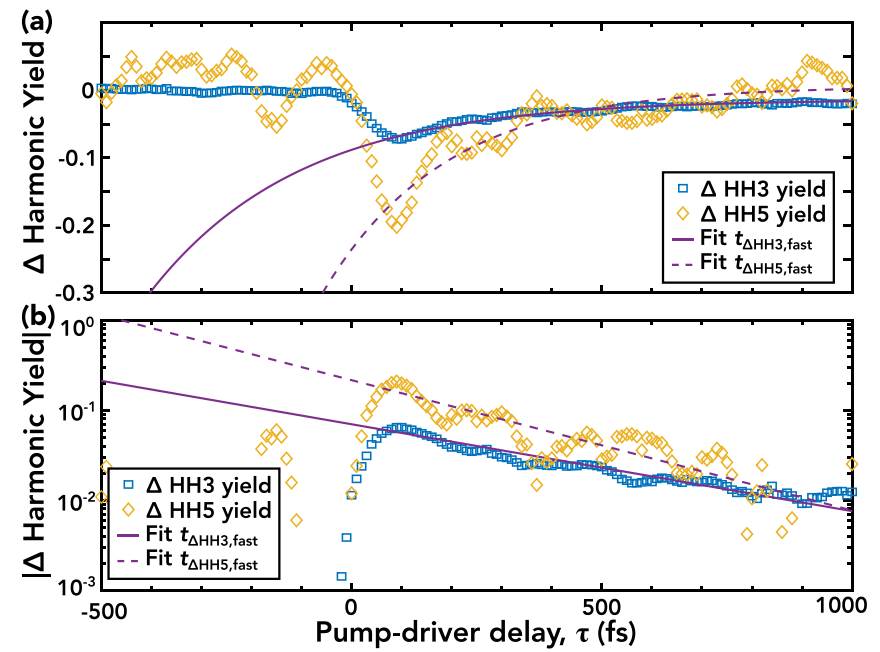

FIG. 7. Fast timescale. (b) Fast time dynamics for the $M_{1}^{*} \rightarrow$ $M_{1}^{*, b}$ transition of $t_{\text {fast }}=300 \pm 24 \mathrm{fs}$ and $245 \pm 68 \mathrm{fs}$ retrieved from the third and fifth tr-HHG measurements respectively. Each measurement was pumped with only $3 \mathrm{~mJ} \mathrm{~cm}^{-2}$ where this is the only transition present. (b) Absolute change in harmonic yield plotted on a semilogarithmic scale to show the single exponential timescale present $\mathrm{HH}$ : high harmonic.

\section{HHG results from this work}

The two timescales present in the change in harmonic yield were each fitted to a biexponential equation of the form

$$
S=A_{0}-A_{1} e^{-\left(t-t_{0, \text { fast }}\right) / t_{c, \text { fast }}}+B_{0}-B_{1} e^{-\left(t-t_{0, \text { slow }}\right) / t_{c, \text { slow }}},
$$

where $t_{c \text {,fast }}$ and $t_{c \text {,slow }}$ are the time constants. This is consistent with the theory analysis of He and Millis [37] who show that the $M_{1} \rightarrow \mathcal{M}$ phase transition has two components: a fast thermalization transition $\left(M_{1}^{*} \rightarrow M_{1}^{*, b}\right)$, followed by the long relaxation to the $\mathcal{M}$ phase $\left(M_{1}^{*, b} \rightarrow \mathcal{M}\right)$. These two transitions are probed using the tr-HHG yield.

The time constants for each transition are found as follows: $M_{1}^{*} \rightarrow M_{1}^{*, b}$ phase transition. The $M_{1}^{*} \rightarrow M_{1}^{*, b}$ time constant is found when the system is pumped by a low fluence of $3 \mathrm{~mJ} \mathrm{~cm}^{-2}$ (at $1.5 \mu \mathrm{m}$ ). At this fluence, there is insufficient energy to initiate the phase transition and the system returns to the $M_{1}$ state after photoexcitation. We fit these features to the third and fifth tr-HHG curves to give us $t_{\Delta \mathrm{HH} 3 \text {, fast }}=300 \pm$ $24 \mathrm{fs}$ and $t_{\triangle \mathrm{HH} 5 \text {,fast }}=245 \pm 68 \mathrm{fs}$ (Fig. 7) respectively, which is in good agreement of the $\sim 100$ s fs found by He and Millis [37].

$M_{1}^{*, b} \rightarrow \mathcal{M}$ phase transition. Looking at an IMT pumped with moderate fluence $\left(12-20 \mathrm{~mJ} \mathrm{~cm}^{-2}\right.$ at $\left.1.5 \mu \mathrm{m}\right)$, we are able to clearly see the $M_{1}^{*, b} \rightarrow \mathcal{M}$ timescales from our datasets (Fig. 8 green curves) and are in good agreement with the UED results of Ref. [35] (Fig. 6). We can extract a slow timescale for the $M_{1}^{*, b} \rightarrow \mathcal{M}$ transition of $t_{\Delta \mathrm{HH} 3 \text {, slow }}=$ $1.45 \pm 0.34$ ps and $t_{\Delta \mathrm{HH} 5 \text {, slow }}=1.10 \pm 0.37 \mathrm{ps}$ for the trHHG yields of the third and fifth harmonics. Figure $8(\mathrm{~b})$ shows the absolute change in harmonic yield plotted on a semilogarithmic scale. In this case, the two timescales present as two intersecting linear fit lines, whose slopes correspond to the timescales of the phase transitions.

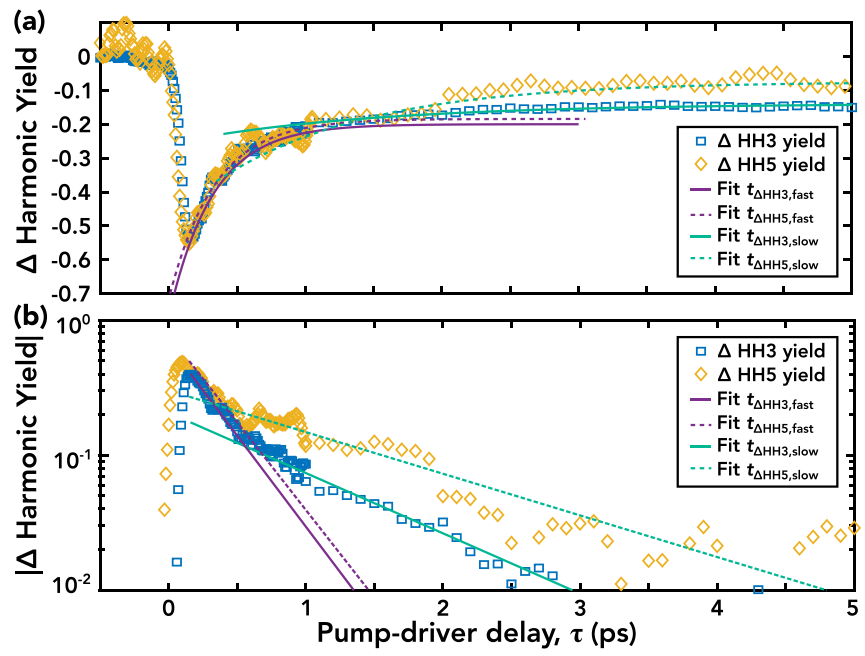

FIG. 8. Two timescales. (a) Initiating the IMT with moderate pump fluences of 20 and $12 \mathrm{~mJ} \mathrm{~cm}^{-2}$ (at $1.5 \mu \mathrm{m}$ ) (HH3, blue squares and HH5, yellow diamonds respectively) presents both the $M_{1}^{*} \rightarrow M_{1}^{*, b}$ and $M_{1}^{*, b} \rightarrow \mathcal{M}$ timescales in the changing harmonic yield for both the third and fifth tr-HHG measurements. Fast dynamics of $t_{\text {fast }}=322 \pm 34$ fs and $400 \pm 108$ fs (purple curves), and slow dynamics of $t_{\text {slow }}=1.45 \pm 0.34 \mathrm{ps}$ and $1.10 \pm 0.37 \mathrm{ps}$ (green curves) are retrieved from the third and fifth harmonic curves respectively. (b) Absolute change in harmonic yield plotted on a semilogarithmic scale showing the biexponential fit as two intersecting linear lines corresponding to timescales in the phase transition HH: high harmonic.

\section{APPENDIX D: THEORETICAL TR-HHG CALCULATIONS FOR SEMICONDUCTOR SYSTEMS}

To demonstrate that the tr-HHG response in $\mathrm{VO}_{2}$ differs dramatically from that expected from a conventional semiconductor system where correlations are negligible, we simulated the tr-HHG response of a model semiconductor with the same band gap $(0.68 \mathrm{eV})$ as $\mathrm{VO}_{2}$. We constructed the tight binding Hamiltonian of a 2D, two-band hexagonal system with a separation between atoms of $a=4.9 \AA$, first neighbor hopping $t=0.1$ a.u., and no higher order hoppings. Following the method described in [61], we used the density matrix equations to propagate the fully filled valence band in the presence of a pump and driver field with the same parameters as in the experiment, i.e., $1.5 \mu \mathrm{m}$ pump of 50 fs duration and intensity of $I=0.3 \mathrm{TW} \mathrm{cm} \mathrm{cm}^{-2}$, and 7 and $10 \mu \mathrm{m}$ drivers of 80 fs duration and intensity $I=2 \mathrm{TW} \mathrm{cm}^{-2}$. We included a dephasing parameter of $T_{2}=4 \mathrm{fs}$, similar to that used before [21]. The HHG response was computed from the current, following the method described in [61].

The left column of Fig. 9 shows the intensity of the HHG spectra at various pump-driver time delays for both $10 \mu \mathrm{m}$ [Fig. 9(a)] and $7 \mu \mathrm{m}$ [Fig. 9(c)] drivers. The right column shows the integrated intensity of the third, fifth, and seventh harmonics as a function of the pump-driver time delay, analogous to that plotted in Fig. 3. The integration was performed over an energy window of $[(N-0.5) \omega,(N+0.5) \omega]$, where $N$ is the harmonic order and $\omega$ is the driver frequency.

The differences between the tr-HHG response in the conventional semiconductor (Fig. 9) and $\mathrm{VO}_{2}$ (Fig. 3) are 

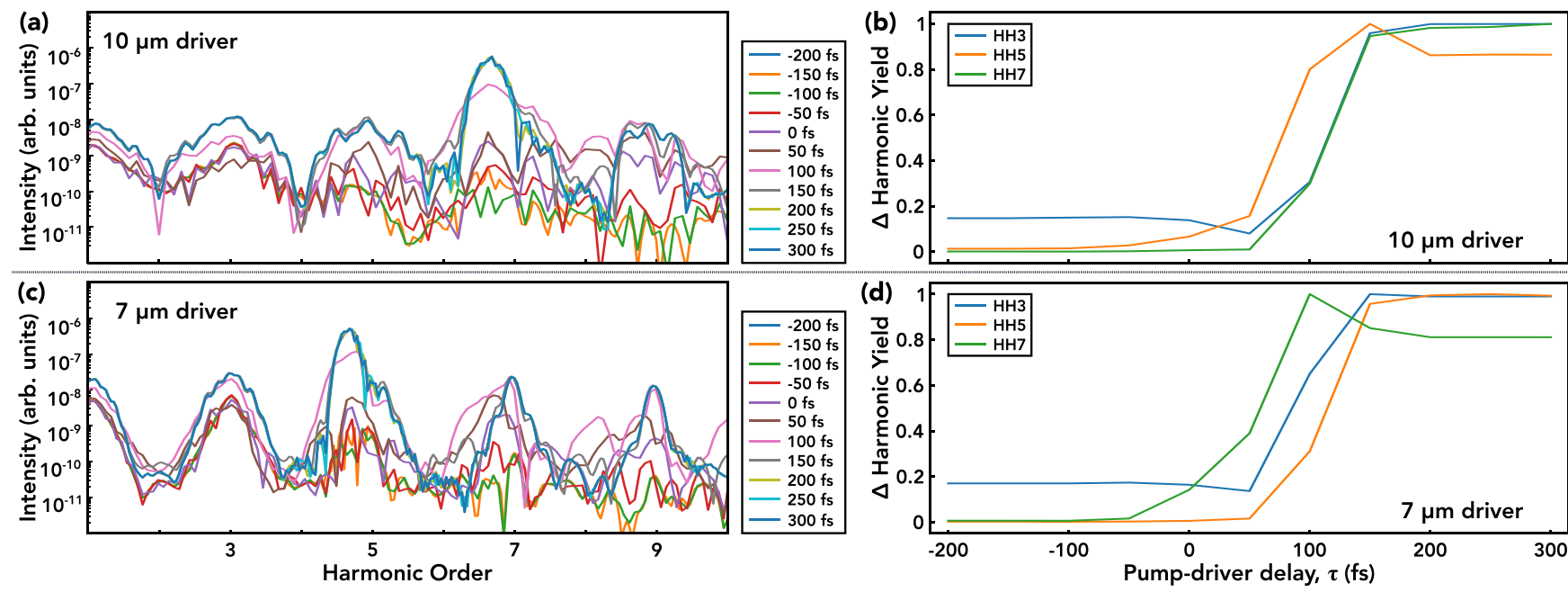

FIG. 9. Calculated tr-HHG traces. Calculated tr-HHG traces for a semiconductor system with a band gap of $0.68 \mathrm{eV}$ photoexcited with $1.5 \mu \mathrm{m}$, and with HHG driven by $10 \mu \mathrm{m}$ (top) or $7 \mu \mathrm{m}$ (bottom). Negative delays indicate the harmonic generating driver pulse arrives before photoexcitation. (a) and (c) show the harmonic spectra for various pump delays. (b) and (d) show the tr-HHG traces for the third, fifth, and seventh harmonic orders for each driver frequency. An increase in harmonic yield is observed for positive delays in this semiconductor system, contrasting with the decrease in yield observed for $\mathrm{VO}_{2}$.

dramatic. For the model conventional semiconductor, where electron correlation is absent, harmonics show an increasing yield when the driver arrives after the pump. This is because the electron population in the conduction band, excited by the pump, concentrates in a specific region of the Brillouin zone. The delayed driver then induces charge oscillations in the $k$ space, generating harmonics. When the pump and the driver pulses overlap, the harmonic yield increases for increasing delay simply because there are more electrons in the conduction band, injected by the pump. These electrons can then be driven to generate intraband harmonics [Figs. 9(b) and 9(d), positive delays]. The harmonic yield reaches a stable value when the pump and the driver fields no longer overlap ( $200 \mathrm{fs})$. This stable value and the rise of the harmonic yield naturally occur in the absence of correlations, because there is no structural modification of the lattice and the system remains a gapped semiconductor for all delays.

The observed dynamics in $\mathrm{VO}_{2}$ are very different as a consequence of the correlated processes. The IMT phase transition and the collapse of the band gap within 25 fs [41] are reflected in the drop in the harmonic yield observed in the experimental tr-HHG traces for $\mathrm{VO}_{2}$ (Fig. 3), as explained in the main text.

A theoretical description of the experiment requires fully correlated, time-dependent simulations of $\mathrm{VO}_{2}$ in the presence of strong laser fields, including a dynamical description of phonons. Such simulations are currently a major theoretical challenge and are out of the scope of this paper, but we point out that several works are currently addressing this problem. Studies have been performed on a 1D Hubbard model [23,24], and $\mathrm{TDDFT}+U$ (time dependent density functional theory plus Hubbard $U$ ) calculations have been extended to incorporate a dynamically modulated Hubbard parameter $U$, where $U$ is modified by the strong electric field [25,62]. More recently, a work was developed in the 1D Hubbard model including a time-dependent description of one phonon mode [63]. These works show promise that these types of experiments could be theoretically simulated in the near future.

\section{APPENDIX E: PHONON MODES}

A fast Fourier transform (FFT) was performed on the trHHG traces showing significant oscillations. These include

(i) $3 \mathrm{rd}$ harmonic of $7 \mu \mathrm{m}, 24 \mathrm{~mJ} \mathrm{~cm}^{-2}$,

(ii) 3rd harmonic of $7 \mu \mathrm{m}, 30 \mathrm{~mJ} \mathrm{~cm}^{-2}$,

(iii) 5 th harmonic of $10 \mu \mathrm{m}, 6 \mathrm{~mJ} \mathrm{~cm}^{-2}$,

(iv) 5 th harmonic of $10 \mu \mathrm{m}, 24 \mathrm{~mJ} \mathrm{~cm}^{-2}$.
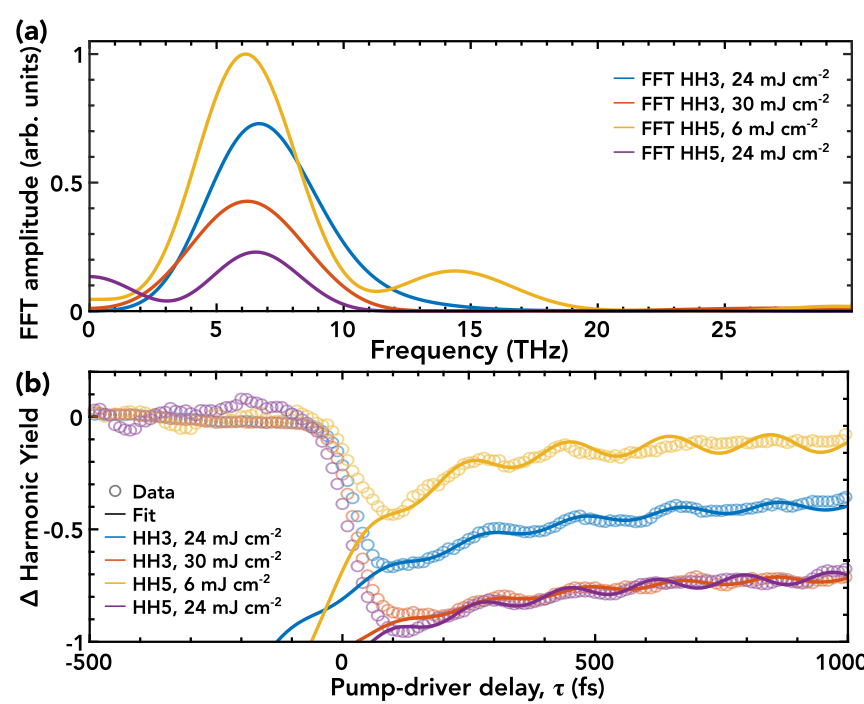

FIG. 10. Phonon mode analysis. (a) Fast Fourier transform of the oscillations observed in Fig. 4(b). (b) Oscillation frequencies retrieved from (a) added to the fast timescales found in Appendix C (solid curves) are overlaid with the tr-HHG data (circles). 


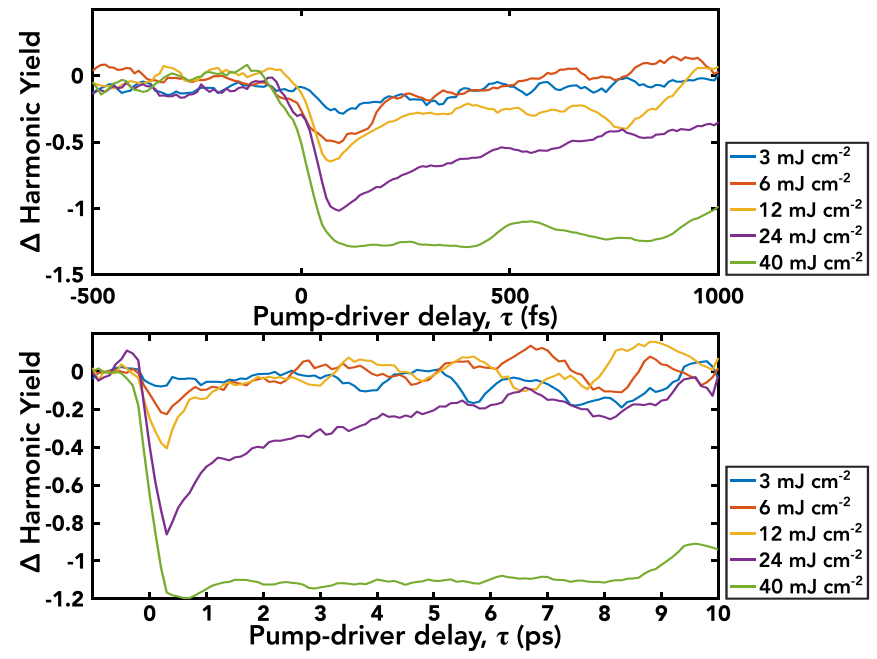

FIG. 11. Harmonic yield in the backwards direction. These curves show the change in harmonic yield for harmonics generated in the backwards direction. We can still see the same double timescale that is observed in transmission.

For these traces, the fast timescale fit found in Appendix C was subtracted for short timescale traces [e.g., Figs. 3(d) and 4(b)] leaving a flat trace with only the phonon oscillation. We then performed an FFT on these traces as shown in Fig. 10(a), revealing peaks at around $6 \pm 2 \mathrm{THz}$. This error encompasses the two lowest order phonon modes at 4.4 and $5.7 \mathrm{THz}$ $[40,47,48,54]$. The large error is due to the low signal-to-noise ratio of our data from the $50 \mathrm{~Hz}$ repetition rate of our laser system. Oscillations with frequencies centered at the peaks found from the FFT were added back to the fast timescale fit from Appendix C, and overlaid with the tr-HHG data in Fig. 10(b) (data: circles; fits: solid curves).

\section{APPENDIX F: BACKWARD DIRECTION HARMONIC MEASUREMENTS}

While the measurements presented in the main text were performed in transmission, the same tr-HHG measurements for the fifth harmonic of $10 \mu \mathrm{m}$ were realized in the backward direction [60], demonstrating the same behavior with a recovery of the harmonic yield for low and intermediate pump fluences (Fig. 11).

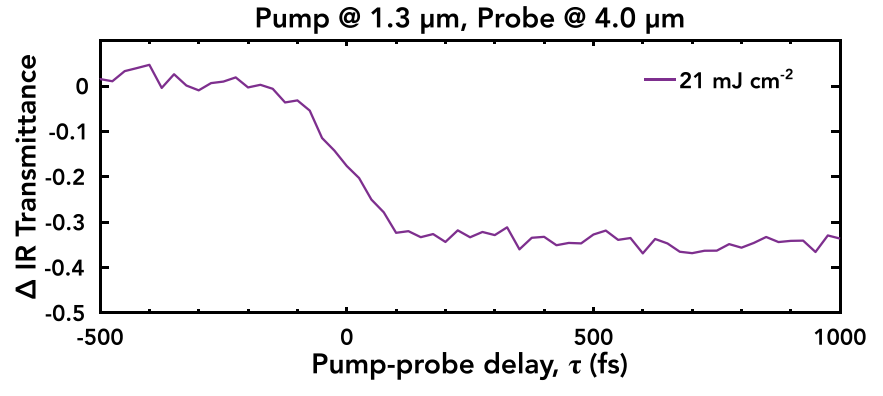

FIG. 12. $1.3 \mu \mathrm{m}$ pump, $4.0 \mu \mathrm{m}$ probe. Complementary measurements with a $1.3 \mu \mathrm{m}$ pump and $4.0 \mu \mathrm{m}$ probe show similar dynamics to the IR transmissivity results presented in the paper.

Backward emitted harmonics are generated on the front surface of the material without propagation through the sample, thus the evolution of the harmonic yield detected originates from variations in the material state. When modulating the pump fluence initiating the IMT, we see the same temporal yield dependency in the backward emitted harmonic generation as in the forward direction. This indicates that the changing of states of $\mathrm{VO}_{2}$ actually affects the production of the harmonics, and does not simply change the transmission of the harmonics through the sample.

As we are able to detect harmonics that are generated in reflection, we can establish that the harmonics are generated on the surface of the sample. Although the signal-to-noise ratio is lower in reflection as there is less signal to collect, the recovery in harmonic yield to the $\mathcal{M}$ state is clearly present alongside the two timescales for transition from the $M_{1}^{*} \rightarrow M_{1}^{*, b}$ and $M_{1}^{*, b} \rightarrow \mathcal{M}$ phases.

\section{APPENDIX G: PUMP-PROBE RESULTS AT OTHER WAVELENGTHS}

Complementary data for IR transmissivity results was compared in the case of a $1.3 \mu \mathrm{m}$ pump and $4.0 \mu \mathrm{m}$ probe (Fig. 12). Although only one fluence was tested, no features were observed besides the expected monotonic drop in transmission. This is similar to the dynamics observed for the $1.5 \mu \mathrm{m}$ pump, and $1.7 \mu \mathrm{m}$ probe presented in the manuscript.
[1] M. Buzzi, M. Först, R. Mankowsky, and A. Cavalleri, Probing dynamics in quantum materials with femtosecond X-rays, Nat. Rev. Mater. 3, 299 (2018).

[2] R. D. Miller, Mapping atomic motions with ultrabright electrons: The chemists' gedanken experiment enters the lab frame, Annu. Rev. Phys. Chem. 65, 583 (2014).

[3] P. Peng, C. Marceau, and D. M. Villeneuve, Attosecond imaging of molecules using high harmonic spectroscopy, Nat. Rev. Phys. 1, 144 (2019).

[4] L. Young, K. Ueda, M. Gühr, P. H. Bucksbaum, M. Simon, S. Mukamel, N. Rohringer, K. C. Prince, C. Masciovecchio, M. Meyer, A. Rudenko, D. Rolles, C. Bostedt, M. Fuchs,
D. A. Reis, R. Santra, H. Kapteyn, M. Murnane, H. Ibrahim, F. Légaré, M. Vrakking, M. Isinger, D. Kroon, M. Gisselbrecht, A. L'Huillier, H. J. Wörner, and S. R. Leone, Roadmap of ultrafast X-ray atomic and molecular physics, J. Phys. B At. Mol. Opt. Phys. 51, 032003 (2018).

[5] R. Geneaux, H. J. Marroux, A. Guggenmos, D. M. Neumark, and S. R. Leone, Transient absorption spectroscopy using high harmonic generation: A review of ultrafast X-ray dynamics in molecules and solids, Philos. Trans. R. Soc. A 377, 20170463 (2019).

[6] A. D. Shiner, B. E. Schmidt, C. Trallero-Herrero, H. J. Wörner, S. Patchkovskii, P. B. Corkum, J.-C. Kieffer, F. Légaré, and D. M. Villeneuve, Probing collective multi-electron dynamics 
in xenon with high-harmonic spectroscopy, Nat. Phys. 7, 464 (2011).

[7] O. Smirnova, Y. Mairesse, S. Patchkovskii, N. Dudovich, D. Villeneuve, P. Corkum, and M. Y. Ivanov, High harmonic interferometry of multi-electron dynamics in molecules, Nature (London) 460, 972 (2009).

[8] P. M. Kraus, B. Mignolet, D. Baykusheva, A. Rupenyan, L. Horný, E. F. Penka, G. Grassi, O. I. Tolstikhin, J. Schneider, F. Jensen, L. B. Madsen, A. D. Bandrauk, F. Remacle, and H. J. Wörner, Measurement and laser control of attosecond charge migration in ionized iodoacetylene, Science 350, 790 (2015).

[9] H. J. Wörner, J. B. Bertrand, D. V. Kartashov, P. B. Corkum, and D. M. Villeneuve, Following a chemical reaction using highharmonic interferometry, Nature (London) 466, 604 (2010).

[10] H. Ruf, C. Handschin, A. Ferré, N. Thiré, J. B. Bertrand, L. Bonnet, R. Cireasa, E. Constant, P. B. Corkum, D. Descamps, B. Fabre, P. Larregaray, E. Mével, S. Petit, B. Pons, D. Staedter, H. J. Wörner, D. M. Villeneuve, Y. Mairesse, P. Halvick, and V. Blanchet, High-harmonic transient grating spectroscopy of $\mathrm{NO}_{2}$ electronic relaxation, J. Chem. Phys. 137, 224303 (2012).

[11] H. J. Wörner, J. B. Bertrand, B. Fabre, J. Higuet, H. Ruf, A. Dubrouil, S. Patchkovskii, M. Spanner, Y. Mairesse, V. Blanchet, E. Mével, E. Constant, P. B. Corkum, and D. M. Villeneuve, Conical intersection dynamics in $\mathrm{NO}_{2}$ probed by homodyne high-harmonic spectroscopy, Science 334, 208 (2011).

[12] S. Ghimire, A. D. Dichiara, E. Sistrunk, P. Agostini, L. F. Dimauro, and D. A. Reis, Observation of high-order harmonic generation in a bulk crystal, Nat. Phys. 7, 138 (2011).

[13] G. Vampa, T. J. Hammond, N. Thiré, B. E. Schmidt, F. Légaré, C. R. McDonald, T. Brabec, and P. B. Corkum, Linking high harmonics from gases and solids, Nature (London) 522, 462 (2015).

[14] M. Hohenleutner, F. Langer, O. Schubert, M. Knorr, U. Huttner, S. W. Koch, M. Kira, and R. Huber, Real-time observation of interfering crystal electrons in high-harmonic generation, Nature (London) 523, 572 (2015).

[15] Z. Wang, H. Park, Y. H. Lai, J. Xu, C. I. Blaga, F. Yang, P. Agostini, and L. F. DiMauro, The roles of photo-carrier doping and driving wavelength in high harmonic generation from a semiconductor, Nat. Commun. 8, 1686 (2017).

[16] M. Baudisch, A. Marini, J. D. Cox, T. Zhu, F. Silva, S. Teichmann, M. Massicotte, F. Koppens, L. S. Levitov, F. J. García De Abajo, and J. Biegert, Ultrafast nonlinear optical response of Dirac fermions in graphene, Nat. Commun. 9, 1018 (2018).

[17] G. Vampa, T. J. Hammond, N. Thiré, B. E. Schmidt, F. Légaré, C. R. McDonald, T. Brabec, D. D. Klug, and P. B. Corkum, AllOptical Reconstruction of Crystal Band Structure, Phys. Rev. Lett. 115, 193603 (2015).

[18] A. A. Lanin, E. A. Stepanov, A. B. Fedotov, and A. M. Zheltikov, Mapping the electron band structure by intraband high-harmonic generation in solids, Optica 4, 516 (2017).

[19] S. Ghimire and D. A. Reis, High-harmonic generation from solids, Nat. Phys. 15, 10 (2019).

[20] D. Golde, T. Meier, and S. W. Koch, High harmonics generated in semiconductor nanostructures by the coupled dynamics of optical inter- and intraband excitations, Phys. Rev. B 77, 075330 (2008).
[21] G. Vampa, C. R. McDonald, G. Orlando, D. D. Klug, P. B. Corkum, and T. Brabec, Theoretical Analysis of HighHarmonic Generation in Solids, Phys. Rev. Lett. 113, 073901 (2014).

[22] G. Vampa and T. Brabec, Merge of high harmonic generation from gases and solids and its implications for attosecond science, J. Phys. B At. Mol. Opt. Phys. 50, 083001 (2017).

[23] R. E. Silva, I. V. Blinov, A. N. Rubtsov, O. Smirnova, and M. Ivanov, High-harmonic spectroscopy of ultrafast many-body dynamics in strongly correlated systems, Nat. Photonics 12, 266 (2018).

[24] Y. Murakami, M. Eckstein, and P. Werner, High-Harmonic Generation in Mott Insulators, Phys. Rev. Lett. 121, 057405 (2018).

[25] N. Tancogne-Dejean, M. A. Sentef, and A. Rubio, Ultrafast Modification of Hubbard $U$ in a Strongly Correlated Material: Ab Initio High-Harmonic Generation in NiO, Phys. Rev. Lett. 121, 097402 (2018).

[26] S. Imai, A. Ono, and S. Ishihara, High Harmonic Generation In A Correlated Electron System, Phys. Rev. Lett. 124, 157404 (2020).

[27] D. N. Basov, R. D. Averitt, and D. Hsieh, Towards properties on demand in quantum materials, Nat. Mater. 16, 1077 (2017).

[28] M. Mitrano, A. Cantaluppi, D. Nicoletti, S. Kaiser, A. Perucchi, S. Lupi, P. Di Pietro, D. Pontiroli, M. Riccò, S. R. Clark, D. Jaksch, and A. Cavalleri, Possible light-induced superconductivity in $\mathrm{K}_{3} \mathrm{C}_{6} \mathrm{O}$ at high temperature, Nature (London) 530, 461 (2016).

[29] T. Oka and S. Kitamura, Floquet engineering of quantum materials, Annu. Rev. Condens. Matter Phys. 10, 387 (2018).

[30] B. Smit, F. Hüwe, N. Payne, O. Olaoye, I. Bauer, J. Pflaum, M. Schwoerer, and H. Schwoerer, Ultrafast pathways of the photoinduced insulator-metal transition in a low-dimensional organic conductor, Adv. Mater. 31, 1900652 (2019).

[31] T. Frigge, B. Hafke, T. Witte, B. Krenzer, C. Streubühr, A. Samad Syed, V. Mikšić Trontl, I. Avigo, P. Zhou, M. Ligges, D. Von Der Linde, U. Bovensiepen, M. Horn-Von Hoegen, S. Wippermann, A. Lücke, S. Sanna, U. Gerstmann, and W. G. Schmidt, Optically excited structural transition in atomic wires on surfaces at the quantum limit, Nature (London) 544, 207 (2017).

[32] A. Zong, A. Kogar, Y. Q. Bie, T. Rohwer, C. Lee, E. Baldini, E. Ergeçen, M. B. Yilmaz, B. Freelon, E. J. Sie, H. Zhou, J. Straquadine, P. Walmsley, P. E. Dolgirev, A. V. Rozhkov, I. R. Fisher, P. Jarillo-Herrero, B. V. Fine, and N. Gedik, Evidence for topological defects in a photoinduced phase transition, Nat. Phys. 15, 27 (2019).

[33] S. Vogelgesang, G. Storeck, J. G. Horstmann, T. Diekmann, M. Sivis, S. Schramm, K. Rossnagel, S. Schäfer, and C. Ropers, Phase ordering of charge density waves traced by ultrafast lowenergy electron diffraction, Nat. Phys. 14, 184 (2018).

[34] J. N. Clark, L. Beitra, G. Xiong, A. Higginbotham, D. M. Fritz, H. T. Lemke, D. Zhu, M. Chollet, G. J. Williams, M. Messerschmidt, B. Abbey, R. J. Harder, A. M. Korsunsky, J. S. Wark, and I. K. Robinson, Ultrafast three-dimensional imaging of lattice dynamics in individual gold nanocrystals, Science 341, 56 (2013).

[35] V. R. Morrison, R. P. Chatelain, K. L. Tiwari, A. Hendaoui, A. Bruhács, M. Chaker, and B. J. Siwick, A photoinduced metallike phase of monoclinic $\mathrm{VO}_{2}$ revealed by ultrafast electron diffraction, Science 346, 455 (2014). 
[36] M. R. Otto, L. P. R. de Cotret, D. A. Valverde-Chavez, K. L. Tiwari, N. Émond, M. Chaker, D. G. Cooke, and B. J. Siwick, How optical excitation controls the structure and properties of vanadium dioxide, Proc. Natl. Acad. Sci. USA 116, 450 (2019).

[37] Z. He and A. J. Millis, Photoinduced phase transitions in narrow-gap Mott insulators: The case of $\mathrm{VO}_{2}$, Phys. Rev. B 93, 115126 (2016).

[38] M. R. Bionta, V. Wanie, V. Gruson, J. Chaillou, N. Émond, D. Lepage, P. Lassonde, M. Chaker, and F. Légaré, Probing the phase transition in $\mathrm{VO}_{2}$ using few-cycle $1.8 \mu \mathrm{m}$ pulses, Phys. Rev. B 97, 125126 (2018).

[39] F. J. Morin, Oxides which Show a Metal-to-Insulator Transition at the Neel Temperature, Phys. Rev. Lett. 3, 34 (1959).

[40] D. Wegkamp and J. Stähler, Ultrafast dynamics during the photoinduced phase transition in $\mathrm{VO}_{2}$, Prog. Surf. Sci. 90, 464 (2015).

[41] M. F. Jager, C. Ott, P. M. Kraus, C. J. Kaplan, W. Pouse, R. E. Marvel, R. F. Haglund, D. M. Neumark, and S. R. Leone, Tracking the insulator-to-metal phase transition in $\mathrm{VO}_{2}$ with few-femtosecond extreme UV transient absorption spectroscopy, Proc. Natl. Acad. Sci. USA 114, 9558 (2017).

[42] S. Wall, S. Yang, L. Vidas, M. Chollet, J. M. Glownia, M. Kozina, T. Katayama, T. Henighan, M. Jiang, T. A. Miller, D. A. Reis, L. A. Boatner, O. Delaire, and M. Trigo, Ultrafast disordering of vanadium dimers in photoexcited $\mathrm{VO}_{2}$, Science 362, 572 (2018).

[43] P. Baum, D. S. Yang, and A. H. Zewail, 4D visualization of transitional structures in phase transformations by electron diffraction, Science 318, 788 (2007).

[44] A. Cavalleri, C. Tóth, C. W. Siders, J. A. Squier, F. Ráksi, P. Forget, and J. C. Kieffer, Femtosecond Structural Dynamics in $\mathrm{VO}_{2}$ during an Ultrafast Solid-Solid Phase Transition, Phys. Rev. Lett. 87, 237401 (2001).

[45] A. Cavalleri, T. Dekorsy, H. H. W. Chong, J. C. Kieffer, and R. W. Schoenlein, Evidence for a structurally-driven insulatorto-metal transition in $\mathrm{VO}_{2}$ : A view from the ultrafast timescale, Phys. Rev. B 70, 161102(R) (2004).

[46] C. Kübler, H. Ehrke, R. Huber, R. Lopez, A. Halabica, R. F. Haglund, and A. Leitenstorfer, Coherent Structural Dynamics and Electronic Correlations during an Ultrafast Insulator-toMetal Phase Transition in $\mathrm{VO}_{2}$, Phys. Rev. Lett. 99, 116401 (2007).

[47] S. Wall, D. Wegkamp, L. Foglia, K. Appavoo, J. Nag, R. F. Haglund, J. StäCurrency Signhler, and M. Wolf, Ultrafast changes in lattice symmetry probed by coherent phonons, Nat. Commun. 3, 721 (2012).

[48] S. Wall, L. Foglia, D. Wegkamp, K. Appavoo, J. Nag, R. F. Haglund, J. Stähler, and M. Wolf, Tracking the evolution of electronic and structural properties of $\mathrm{VO}_{2}$ during the ultrafast photoinduced insulator-metal transition, Phys. Rev. B 87, 115126 (2013).
[49] D. Wegkamp, M. Herzog, L. Xian, M. Gatti, P. Cudazzo, C. L. McGahan, R. E. Marvel, R. F. Haglund, A. Rubio, M. Wolf, and J. Stähler, Instantaneous Band Gap Collapse in Photoexcited Monoclinic $\mathrm{VO}_{2}$ due to Photocarrier Doping, Phys. Rev. Lett. 113, 216401 (2014).

[50] J. K. Clark, Y.-L. Ho, H. Matsui, B. Vilquin, H. Tabata, and J.-J. Delaunay, Photoinduced metal-like phase of $\mathrm{VO}_{2}$ with subns recovery, ACS Photonics 7, 2395 (2020).

[51] W. H. Brito, M. C. O. Aguiar, K. Haule, and G. Kotliar, Metal-Insulator Transition in $\mathrm{VO}_{2}$ : A DFT + DMFT Perspective, Phys. Rev. Lett. 117, 056402 (2016).

[52] V. Eyert, $\mathrm{VO}_{2}$ : A Novel View from Band Theory, Phys. Rev. Lett. 107, 016401 (2011).

[53] F. Grandi, A. Amaricci, and M. Fabrizio, Unraveling the MottPeierls intrigue in vanadium dioxide, Phys. Rev. Research 2 , 013298 (2020).

[54] P. Schilbe, Raman scattering in $\mathrm{VO}_{2}$, Phys. B Condens. Matter 316-317, 600 (2002).

[55] R. Hollinger, V. Shumakova, A. Pugžlys, A. Baltuška, S. Khujanov, C. Spielmann, and D. Kartashov, High-order harmonic generation traces ultrafast coherent phonon dynamics in ZnO, EPJ Web Conf. 205, 02025 (2019).

[56] L. von Grafenstein, M. Bock, D. Ueberschaer, K. Zawilski, P. Schunemann, U. Griebner, and T. Elsaesser, $5 \mu \mathrm{m}$ few-cycle pulses with multi-gigawatt peak power at a $1 \mathrm{kHz}$ repetition rate, Opt. Lett. 42, 3796 (2017).

[57] A. Leblanc, P. Lassonde, S. Petit, J.-C. Delagnes, E. Haddad, G Ernotte, M. R. Bionta, V. Gruson, B. E. Schmidt, H. Ibrahim, E. Cormier, and F. Légaré, Phase-matching-free pulse retrieval based on transient absorption in solids, Opt. Express 27, 28998 (2019).

[58] S. Ghimire, G. Ndabashimiye, A. D. Dichiara, E. Sistrunk, M. I. Stockman, P. Agostini, L. F. Dimauro, and D. A. Reis, Strongfield and attosecond physics in solids, J. Phys. B At. Mol. Opt. Phys. 47, 204030 (2014).

[59] L. Keldysh, Ionization in a field of a strong electromagnetic wave, J. Exptl. Theor. Phys. (U.S.S.R.) 47, 1945 (1965) [Sov. Phys. JETP 20, 1307 (1965)].

[60] G. Vampa, Y. S. You, H. Liu, S. Ghimire, and D. A. Reis, Observation of backward high-harmonic emission from solids, Opt. Express 26, 12210 (2018).

[61] R. E. F. Silva, F. Martín, and M. Ivanov, High harmonic generation in crystals using maximally localized Wannier functions, Phys. Rev. B 100, 195201 (2019).

[62] N. Tancogne-Dejean, M. J. T. Oliveira, and A. Rubio, Selfconsistent DFT $+U$ method for real-space time-dependent density functional theory calculations, Phys. Rev. B 96, 245133 (2017).

[63] F. Grandi, J. Li, and M. Eckstein, Ultrafast Mott transition driven by nonlinear electron-phonon interaction, Phys. Rev. B 103, L041110 (2021). 